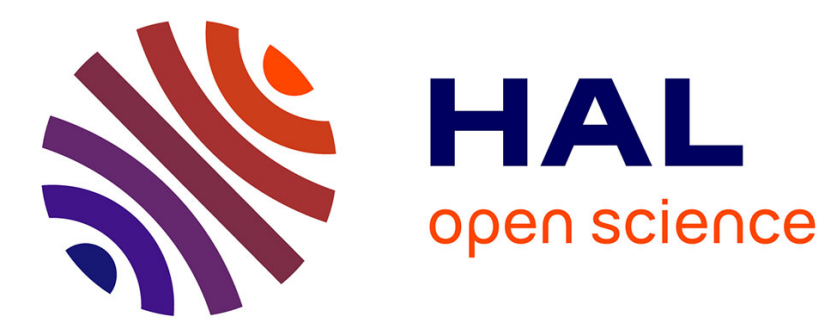

\title{
Hopf-flip bifurcations of vibratory systems with impacts G.W. Luo
}

\section{To cite this version:}

G.W. Luo. Hopf-flip bifurcations of vibratory systems with impacts. Nonlinear Analysis: Real World Applications, 2006, 7 (5), pp.1029-1041. 10.1016/j.nonrwa.2005.09.004 . hal-01352124

\section{HAL Id: hal-01352124 \\ https://hal.science/hal-01352124}

Submitted on 5 Aug 2016

HAL is a multi-disciplinary open access archive for the deposit and dissemination of scientific research documents, whether they are published or not. The documents may come from teaching and research institutions in France or abroad, or from public or private research centers.
L'archive ouverte pluridisciplinaire HAL, est destinée au dépôt et à la diffusion de documents scientifiques de niveau recherche, publiés ou non, émanant des établissements d'enseignement et de recherche français ou étrangers, des laboratoires publics ou privés.

\section{(c)(1)}

Distributed under a Creative Commons Attribution| 4.0 International License 


\title{
Hopf-flip bifurcations of vibratory systems with impacts
}

\author{
G.W. Luo \\ School of Mathematics, Physics and Software Engineering, Lanzhou Jiaotong University, Lanzhou, 730070, People's Republic of China
}

\begin{abstract}
Two vibro-impact systems are considered. The period $n$ single-impact motions and Poincaré maps of the vibro-impact systems are derived analytically. Stability and local bifurcations of single-impact periodic motions are analyzed by using the Poincaré maps. A center manifold theorem technique is applied to reduce the Poincaré map to a three-dimensional one, and the normal form map associated with Hopf-flip bifurcation is obtained. It is found that near the point of codim 2 bifurcation there exists not only Hopf bifurcation of period one single-impact motion, but also Hopf bifurcation of period two double-impact motion. Period doubling bifurcation of period one single-impact motion is commonly existent near the point of codim 2 bifurcation. However, no period doubling cascade emerges due to change of the type of period two fixed points and occurrence of Hopf bifurcation associated with period two fixed points. The results from simulation shows that there exists an interest torus doubling bifurcation occurring near the value of Hopf-flip bifurcation. The torus doubling bifurcation makes the quasi-periodic attractor associated with period one single-impact motion transit to the other quasi-periodic attractor represented by two attracting closed circles. The torus bifurcation is qualitatively different from the typical torus doubling bifurcation occurring in the vibro-impact systems.
\end{abstract}

Keywords: Vibration; Clearance; Impact map; Periodic motion; Hopf-flip bifurcation

\section{Introduction}

Impact oscillators arise whenever the components of a vibrating system collide with rigid obstacles or with each other. Such systems with repeated impacts exist in a wide variety of engineering applications, particularly in mechanisms and machines with clearances or gaps. The principle of operation of vibration hammers, impact dampers, shakers, pile drivers, machinery for compacting, milling and forming, etc., is based on the impact action for moving bodies. With other equipment, e.g., mechanisms with clearances, heat exchangers, fuel elements of nuclear reactors, gears, piping systems, wheel-rail interaction of high speed railway coaches, etc., impacts also occur, but they are undesirable as they bring about failures, strain, shorter service life and increased noise levels. Researches into repeated impact dynamics have important significance in optimization design of machinery with rigid obstacles or clearances, noise suppression and reliability analyses, etc. The physical process during impacts is of strongly non-linear and discontinuous characteristics. The presence of the non-linearity and discontinuity complicates the dynamic analysis of repeated impact systems considerably, but it can be described theoretically and numerically by discontinuities in good agreement with reality. Compared with single impact, vibro-impact dynamics is more complicated, and hence, has received great 
attention. The large interest in analyzing and understanding the performance of such systems is reflected by vast and ever increasing amount of research effort devoted in this area in recent years, a small sample of which is reported in Refs. [1-6,8-11,14-20,23]. However, these studies focused mainly attention on codim 1 bifurcations of the vibro-impact systems. The purpose of the present study is to focus attention on codim 2 bifurcation of single-impact periodic motion. The results from qualitative analysis and numerical simulation shows that the vibro-impact systems, near the points of codim 2 bifurcations, can exhibit richer and more complicated dynamical behavior.

\section{Mechanical model and Poincaré map}

The mechanical model for a vibro-impact system with masses $M_{1}$ and $M_{2}$ is shown in Fig. 1. The vibro-impact system is similar to the model of the impact-forming machine with double masses (see p. 15). Displacements of the masses $M_{1}$ and $M_{2}$ are represented by $X_{1}$ and $X_{2}$, respectively. The masses are connected to linear springs with stiffnesses $K_{1}$, $K_{2}$ and $K_{3}$, and linear viscous dashpots with damping constants $C_{1}, C_{2}$ and $C_{3}$. The excitations on both masses are harmonic with amplitudes $P_{1}$ and $P_{2}$. Here $\Omega$ is the excitation frequency, and $\tau$ the phase angle. The mass $M_{1}$ impact mutually with the mass $M_{2}$ when the difference of their displacements equals the clearance $\Delta$. The impact is described by the conservation law of momentum and a coefficient of restitution $R$, and it is assumed that the duration of impact is negligible compared to the period of the force.

The motion processes of the system, between any two consecutive impacts, are considered. Between any two consecutive impacts, the time $T$ is always set to zero directly at the instant when the former impact is over, and the phase angle $\tau$ is used only to make a suitable choice for the origin of time in the calculation. The state of the vibro-impact system, immediately after impact, has become initial conditions in the subsequent process of the motion. Between consecutive impacts, the non-dimensional differential equations of motion are given by

$$
\left[\begin{array}{cc}
\mu_{m} & 0 \\
0 & 1
\end{array}\right]\left\{\begin{array}{l}
\ddot{x}_{1} \\
\ddot{x}_{2}
\end{array}\right\}+\left[\begin{array}{cc}
2 \zeta\left(1+\gamma_{1}\right) & -2 \zeta \\
-2 \zeta & 2 \zeta\left(1+\gamma_{3}\right)
\end{array}\right]\left\{\begin{array}{l}
\dot{x}_{1} \\
\dot{x}_{2}
\end{array}\right\}+\left[\begin{array}{cc}
k_{1}+1 & -1 \\
-1 & k_{3}+1
\end{array}\right]\left\{\begin{array}{l}
x_{1} \\
x_{2}
\end{array}\right\}=\left\{\begin{array}{c}
1-f_{20} \\
f_{20}
\end{array}\right\} \sin (\omega t+\tau),
$$

where the non-dimensional quantities

$$
\begin{aligned}
& \mu_{m}=\frac{M_{1}}{M_{2}}, \quad k_{i}=\frac{K_{i}}{K_{2}}, \quad \gamma_{i}=\frac{C_{i}}{C_{2}}, \quad i=1,3, \\
& f_{20}=\frac{P_{2}}{P_{1}+P_{2}}, \quad \omega=\Omega \sqrt{\frac{M_{2}}{K_{2}}}, \quad t=T \sqrt{\frac{K_{2}}{M_{2}}}, \quad \zeta=\frac{C_{2}}{2 \sqrt{K_{2} M_{2}}}, \quad \delta=\frac{\Delta \cdot K_{2}}{P_{1}+P_{2}}, \quad x_{i}=\frac{X_{i} K_{2}}{P_{1}+P_{2}}
\end{aligned}
$$

have been introduced. In Eq. (1) a dot (.) denotes differentiation with respect to the non-dimensional time $t$.

When the impact occurs, for $x_{1}(t)-x_{2}(t)=\delta$, the velocities of the masses $M_{1}$ and $M_{2}$ are changed according to the conservation law of momentum, and the impact equation of both masses and the coefficient of restitution $r$ are

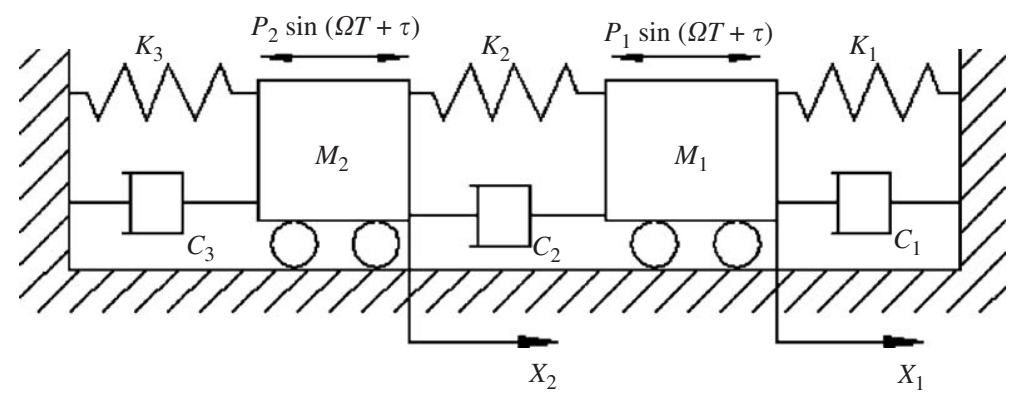

Fig. 1. Schematic of the impact oscillator with double masses and a clearance. 
given by

$$
\mu_{m} \dot{x}_{1+}+\dot{x}_{2+}=\mu_{m} \dot{x}_{1-}+\dot{x}_{2-}, \quad R=\left(\dot{x}_{2+}-\dot{x}_{1+}\right) /\left(\dot{x}_{1-}-\dot{x}_{2-}\right),
$$

where the subscript minus sign denotes the states just before impact and the subscript plus sign denotes the states just after impact, $\dot{x}_{i-}$ and $\dot{x}_{i+}(i=1,2)$ represent the velocities of immediately before and after the impact of the masses $M_{i}$, respectively.

Impacting systems are conveniently studied by use of a mapping derived from the equations of motion. Each iterate of this map corresponds to the mass $M_{1}$ striking the mass $M_{2}$ once. Under suitable system parameter conditions, the system shown in Fig. 1 can exhibit periodic-impact behavior. We can characterize periodic motions of the system by a symbol $q=p / n$, where $p$ is the number of impacts and $n$ is the number of the forcing cycles. The symbol $q$, originated by Peterka, is of important significance in analyzing the periodic-impact motions and bifurcation characteristics, and quantity $q$ has rational and irrational value for periodic and chaotic motions, respectively; see Ref. [19]. Let $\theta=\omega t$, we can choose a Poincaré section $\sigma=\left\{\left(x_{1}, \dot{x}_{1}, x_{2}, \dot{x}_{2}, \theta\right) \in \boldsymbol{R}^{4} \times \boldsymbol{S}, x_{1}-x_{2}=\delta, \dot{x}_{1}=\dot{x}_{1+}, \dot{x}_{2}=\dot{x}_{2+}\right\}$ to establish the Poincaré map of the vibro-impact system. The $q=\frac{1}{1}$ motion and its disturbed map are derived exactly in Ref. [12]. The disturbed map of $q=\frac{1}{1}$ motion is expressed briefly by

$$
X^{\prime}=f(v, X)
$$

where $X \in \boldsymbol{R}^{4}, v \in \boldsymbol{R}^{1}$ or $\boldsymbol{R}^{2} ; X=X^{*}+\Delta X, X^{\prime}=X^{*}+\Delta X^{\prime}, X^{*}=\left(\dot{x}_{1+}, x_{20}, \dot{x}_{2+}, \tau_{0}\right)^{\mathrm{T}}$ is a fixed point in the hyperplane $\sigma, \Delta X$ and $\Delta X^{\prime}$ are the disturbed vectors of $X^{*}$.

Linearizing the Poincaré map at the fixed point $X^{*}$ results in the matrix

$$
\mathrm{D} f\left(v, X^{*}\right)=\left.\frac{\partial f(v, X)}{\partial X}\right|_{\left(v, X^{*}\right)} .
$$

The stability and local bifurcation of $q=\frac{1}{1}$ motion can be analyzed by computing eigenvalues of the linearized matrix $\mathrm{D} f\left(v, X^{*}\right)$ of Poincaré map. If the moduli of all eigenvalues of $\mathrm{D} f\left(v, X^{*}\right)$ are less than one (i.e. $\left.\left|\lambda_{i}(v)\right|<1\right)$, then the corresponding $q=\frac{1}{1}$ motion is stable. In contrast, if the modulus of one of the eigenvalues of $\mathrm{D} f\left(v, X^{*}\right)$ is greater than unity, the fixed point is unstable. In general, bifurcations occur in various ways according to the numbers of the eigenvalues on the unit circle and their positions on the unit circle. In the following sections, Hopf-flip bifurcation of the repeated impact system will be analyzed.

\section{Center manifold and normal form map}

We continue to consider the Poincaré map $X^{\prime}=f(v, X) . X^{*}$ is a fixed point for the map for $v$ in some neighborhood of a critical value $v=v_{\mathrm{c}}$ at which $\mathrm{D} f\left(v, X^{*}\right)$ satisfies the following assumptions:

H1. Jacobian matrix $\mathrm{D} f\left(v, X^{*}\right)$ has the eigenvalues $\lambda_{1}\left(v_{\mathrm{c}}\right)=-1, \lambda_{2}\left(v_{\mathrm{c}}\right)=\bar{\lambda}_{3}\left(v_{\mathrm{c}}\right)$ and $\left|\lambda_{2,3}\left(v_{\mathrm{c}}\right)\right|=1$;

$\mathrm{H} 2$. the remainder of the spectrum of $\mathrm{D} f\left(v, X^{*}\right)$ are strictly inside the unit circle.

For all $v$ in some neighborhood of $v_{\mathrm{c}}$, the map (4), under the change of variables $\mu_{1}=v_{1}-v_{1 \mathrm{c}}, \mu_{2}=v_{2}-v_{2 \mathrm{c}}$, $\mu=\left(\mu_{1}, \mu_{2}\right)^{\mathrm{T}}$ and $X=X^{*}+P \tilde{Y}$, becomes

$$
\tilde{Y}^{\prime}=\tilde{F}(\mu ; \tilde{Y})
$$

where $P$ is the eigenmatrix [7], $\tilde{Y}=\left(y_{1}, y_{2}, y_{3}, y_{4}\right)^{\mathrm{T}}, \tilde{F}=\left(F_{1}, F_{2}, F_{3}, F_{4}\right)^{\mathrm{T}}$.

Let $z_{1}=y_{1}, z_{2}=y_{2}+\mathrm{i} y_{3}, \bar{z}_{2}=y_{2}-\mathrm{i} y_{3}, z=\left(z_{1}, z_{2}, \bar{z}_{2}\right)^{\mathrm{T}}, W=y_{4}, G_{1}=F_{1}-\lambda_{1} z_{1}, G_{2}=F_{2}+\mathrm{i} F_{3}-\lambda_{2} z_{2}$ and $H=F_{4}-\lambda_{4} W$, the map (5) is expressed by

$$
\begin{aligned}
& z_{1}^{\prime}=\lambda_{1} z_{1}+G_{1}\left(z_{1}, z_{2}, \bar{z}_{2}, W ; \mu\right), \quad z_{2}^{\prime}=\lambda_{2} z_{2}+G_{2}\left(z_{1}, z_{2}, \bar{z}_{2}, W ; \mu\right), \\
& W^{\prime}=\lambda_{4} W+H\left(z_{1}, z_{2}, \bar{z}_{2}, W ; \mu\right),
\end{aligned}
$$


in which $\lambda_{1}=\tilde{\lambda}_{1}(\mu)=\lambda_{1}\left(v_{\mathrm{c}}+\mu\right), \lambda_{2}=\tilde{\lambda}_{2}(\mu)=\lambda_{2}\left(v_{\mathrm{c}}+\mu\right), \lambda_{1}(0)=-1, \lambda_{2,3}(0)=\alpha \pm \mathrm{i} \varpi,\left|\lambda_{2,3}(0)\right|=1$. For the map (6), there exists a local center manifold $W\left(z_{1}, z_{2}, \bar{z}_{2} ; \mu\right)$, which can be determined by the equation

$$
W\left(z_{1}^{\prime}, z_{2}^{\prime}, \bar{z}_{2}^{\prime} ; \mu\right)=\lambda_{4} W\left(z_{1}, z_{2}, \bar{z}_{2} ; \mu\right)+H\left(z_{1}, z_{2}, \bar{z}_{2}, W\left(z_{1}, z_{2}, \bar{z}_{2} ; \mu\right) ; \mu\right) .
$$

On the center manifold the local behavior of the map (7) can be reduced to a three-dimensional map $\tilde{\Phi}(z ; \mu)$, which is now

$$
z_{1}^{\prime}=\lambda_{1}(\mu) z_{1}+G_{1}\left(z_{1}, z_{2}, \bar{z}_{2}, W\left(z_{1}, z_{2}, \bar{z}_{2} ; \mu\right) ; \mu\right), \quad z_{2}^{\prime}=\lambda_{2}(\mu) z_{2}+G_{2}\left(z_{1}, z_{2}, \bar{z}_{2}, W\left(z_{1}, z_{2}, \bar{z}_{2} ; \mu\right) ; \mu\right),
$$

where the Taylor series expansion of $W\left(z_{1}, z_{2}, \bar{z}_{2} ; \mu\right)$ and $G_{i}\left(z_{1}, z_{2}, \bar{z}_{2}, W\left(z_{1}, z_{2}, \bar{z}_{2} ; \mu\right) ; \mu\right)$ about $(0,0,0, \mu)$ can be determined by a similar method used in Ref. [13].

By using the center manifold theorem technique and normal form method of maps, we can reduce the map (8) to the normal form map $\Phi(z ; \varepsilon)$, which is given by

$$
z_{1}^{\prime}=-z_{1}+\varepsilon_{1} z_{1}+a z_{1}^{3}+b z_{1}\left|z_{2}\right|^{2}+O\left(|z|^{5}\right), \quad z_{2}^{\prime}=\lambda_{2}(0) z_{2}+\tilde{\varepsilon}_{2} z_{2}+\tilde{c} z_{1}^{2} z_{2}+\tilde{d} z_{2}\left|z_{2}\right|^{2}+O\left(|z|^{5}\right) .
$$

The normal form map (9) $\Phi(Y ; \varepsilon)$, in the real form, is expressed by

$$
\left\{\begin{array}{l}
y_{1}^{\prime}=-y_{1}+\varepsilon_{1} y_{1}+a y_{1}^{3}+b y_{1}\left(y_{2}^{2}+y_{3}^{2}\right)+\text { h.o.t } \\
y_{2}^{\prime}=\left(\alpha+\varepsilon_{2}\right) y_{2}-\left(\varpi+\varepsilon_{3}\right) y_{3}+\left(c y_{2}-e y_{3}\right) y_{1}^{2}+\left(d y_{2}-f y_{3}\right)\left(y_{2}^{2}+y_{3}^{2}\right)+\text { h.o.t } \\
y_{3}^{\prime}=\left(\varpi+\varepsilon_{3}\right) y_{2}+\left(\alpha+\varepsilon_{2}\right) y_{3}+\left(c y_{3}+e y_{2}\right) y_{1}^{2}+\left(d y_{3}+f y_{2}\right)\left(y_{2}^{2}+y_{3}^{2}\right)+\text { h.o.t }
\end{array}\right.
$$

in which, $\varepsilon=\left(\varepsilon_{1}, \varepsilon_{2}, \varepsilon_{3}\right)^{\mathrm{T}}, \varepsilon_{i}=\varepsilon_{i}(\mu), \varepsilon_{i}(0)=0$.

\section{Local codim 2 bifurcation of the normal form map}

Let us assume that there exist period two fixed points for the map $\Phi(Y ; \varepsilon)$. In view of the normal form map, the period two points $Y_{1}^{(2)}$ and $Y_{2}^{(2)}$ satisfy the equation

$$
\Phi^{2}\left(Y_{i}^{(2)} ; \varepsilon\right)=Y_{i}^{(2)} .
$$

Ignoring the terms of high order, the solutions of Eq. (11) are

$$
Y_{1}^{(2)}=\left(\sqrt{-\varepsilon_{1} / a}, 0,0\right)^{\mathrm{T}}, \quad Y_{2}^{(2)}=\left(-\sqrt{-\varepsilon_{1} / a}, 0,0\right)^{\mathrm{T}} .
$$

If $\varepsilon_{1} / a<0$, there exist the period two points $Y^{(2)}=\left(Y_{1}^{(2)}, Y_{2}^{(2)}\right)^{\mathrm{T}}$, and they are symmetrical about the origin. The linearized maps of $\Phi(Y ; \varepsilon)$ at the fixed point and $\Phi^{2}(Y ; \varepsilon)$ at the period two points, respectively, are given by

$$
Q_{1}=\left.\frac{\partial \Phi(Y ; \varepsilon)}{\partial Y}\right|_{(0, \varepsilon)}, \quad Q_{2}=\left.\frac{\partial \Phi^{2}(Y ; \varepsilon)}{\partial Y}\right|_{\left(Y_{i}^{(2)}, \varepsilon\right)} .
$$

The partial bifurcation sets for the normal form map can be determined by computing and analyzing the eigenvalues of Jacobian matrices (13). However, a full understanding of the normal form map (10) requires more than Jacobian matrices (13). So it is necessary to change the normal form map to the polar coordinate form $\Phi\left(r, \theta ; \varepsilon_{0}\right) \in \boldsymbol{R}^{2} \times \boldsymbol{S}$, $(x, r, \theta) \rightarrow\left(x^{\prime}, r^{\prime}, \theta^{\prime}\right)$.

Let $\tilde{\varepsilon}_{2}=\tilde{\lambda}_{2}(0) \tilde{\varepsilon}_{20}, \tilde{c}=\tilde{\lambda}_{2}(0) \tilde{c}_{0}, \tilde{d}=\tilde{\lambda}_{2}(0) \tilde{d}_{0}$, the normal form map (9) becomes

$$
\begin{aligned}
& z_{1}^{\prime}=-z_{1}\left(1-\varepsilon_{1}-a z_{1}^{2}-b\left|z_{2}\right|^{2}\right)+O\left(\left(\left|z_{1}\right|+\left|z_{2}\right|\right)^{5}\right), \\
& z_{2}^{\prime}=\tilde{\lambda}_{2}(0) z_{2}\left(1+\tilde{\varepsilon}_{20}+\tilde{c}_{0} z_{1}^{2}+\tilde{d}_{0}\left|z_{2}\right|^{2}\right)+O\left(\left(\left|z_{1}\right|+\left|z_{2}\right|\right)^{5}\right) .
\end{aligned}
$$

In polar coordinates, the map $\Phi\left(z ; \varepsilon_{0}\right) \in \boldsymbol{R}^{3}$ is changed to $\Phi\left(x, r, \theta ; \varepsilon_{0}\right) \in \boldsymbol{R}^{2} \times \boldsymbol{S}$, which is given by

$$
\begin{aligned}
& x^{\prime}=-x\left(1-\varepsilon_{1}-a x^{2}-b r^{2}\right)+\text { h.o.t, } \quad r^{\prime}=r\left(1+\varepsilon_{20}+c_{0} x^{2}+d_{0} r^{2}\right)+\text { h.o.t }, \\
& \theta^{\prime}=\theta+\theta_{0}+\varepsilon_{30}+e_{0} x^{2}+f_{0} r^{2}+\text { h.o.t. }
\end{aligned}
$$


in which $\varepsilon_{20}=\alpha \varepsilon_{2}+\varpi \varepsilon_{3}, \varepsilon_{30}=\alpha \varepsilon_{3}-\varpi \varepsilon_{2}, c_{0}=\alpha c+\varpi e, d_{0}=\alpha d+\varpi f, e_{0}=\alpha e-\varpi c, f_{0}=\alpha f-\varpi d$. We ignore the influence of phase angle $\theta$ to the map (15) temporarily so that a simplified map is obtained, which is

$$
x^{\prime}=-x\left(1-\varepsilon_{1}-a x^{2}-b r^{2}\right)+\text { h.o.t, } \quad r^{\prime}=r\left(1+\varepsilon_{20}+c_{0} x^{2}+d_{0} r^{2}\right)+\text { h.o.t. }
$$

Let the iterated map $\hat{\Phi}^{2}\left(x, r ; \varepsilon_{0}\right)=\left(x^{(2)}, r^{(2)} ; \varepsilon_{0}\right)^{\mathrm{T}}$, then we can write

$$
x^{(2)}=x+2\left(-x \varepsilon_{1}-a x^{3}-b x r^{2}\right)+\text { h.o.t, } \quad r^{(2)}=r+2\left(r \varepsilon_{20}+c_{0} x^{2} r+d_{0} r^{3}\right)+\text { h.o.t. }
$$

The map (16) has two fixed points $Y_{0}=(0,0)^{\mathrm{T}}$ and $Y_{1}^{*}=\left(0, \sqrt{-\varepsilon_{20} / d_{0}}\right)^{\mathrm{T}}$, and the iterated map (17) has two pairs of fixed points $Y_{21}^{*}=\left(\sqrt{-\varepsilon_{1} / a}, 0\right)^{\mathrm{T}}, Y_{22}^{*}=\left(-\sqrt{-\varepsilon_{1} / a}, 0\right)^{\mathrm{T}}$;

$$
Y_{31}^{*}=\left(\sqrt{\frac{b \varepsilon_{20}-d_{0} \varepsilon_{1}}{a d_{0}-b c_{0}}}, \sqrt{\frac{c_{0} \varepsilon_{1}-a \varepsilon_{20}}{a d_{0}-b c_{0}}}\right)^{\mathrm{T}}, \quad Y_{32}^{*}=\left(-\sqrt{\frac{b \varepsilon_{20}-d_{0} \varepsilon_{1}}{a d_{0}-b c_{0}}}, \sqrt{\frac{c_{0} \varepsilon_{1}-a \varepsilon_{20}}{a d_{0}-b c_{0}}}\right)^{\mathrm{T}} .
$$

Three main cases associated with scheme of coefficients of high order terms, i.e.,

Case (I): $a>0, b>0, c_{0}<0, d_{0}<0, a d_{0}-b c_{0}<0, a+c_{0}>0$ and $b+d_{0}>0$;

Case (II): $a>0, b>0, c_{0}>0, d_{0}>0, a d_{0}-b c_{0}<0$;

Case (III): $a<0, b<0, c_{0}<0, d_{0}<0, a d_{0}-b c_{0}<0$, are considered, respectively. The Cases (I) and (II) are called the simple cases; the Case (III) the complex case.

The bifurcation set for the map (16), near $\varepsilon=\left(\varepsilon_{1}, \varepsilon_{20}\right)^{\mathrm{T}}=(0,0)^{\mathrm{T}}$, can be illustrated by Figs. 2(a)-(c). Only the positive $(x, r)$ quadrant is shown in Figs. 2(a)-(c). Since the portraits are symmetric under reflection about $r$ axis. The bounds of the regions, shown in Figs. 2(a) and (b), can be listed as follows:

$$
\begin{aligned}
& L_{1}: \varepsilon_{1}=0, \varepsilon_{20}<0 ; L_{2}: \varepsilon_{20}=\frac{c_{0}}{a} \varepsilon_{1}, \varepsilon_{1}<0 ; L_{3}: \varepsilon_{20}=\frac{d_{0}\left(a+c_{0}\right)}{a\left(d_{0}+b\right)} \varepsilon_{1}, \varepsilon_{1}<0 ; \\
& L_{4}: \varepsilon_{20}=0, \varepsilon_{1}>0 ; L_{5}: \varepsilon_{20}=\frac{d_{0}}{b} \varepsilon_{1}, \varepsilon_{1}<0 .
\end{aligned}
$$

The bounds of the regions, shown in Fig. 2(c), can be listed as follows

$$
\begin{aligned}
& L_{1}: \varepsilon_{1}=0, \varepsilon_{20}<0 ; L_{2}: \varepsilon_{20}=0, \varepsilon_{1}>0 ; L_{3}: \varepsilon_{20}=\frac{d_{0}}{b} \varepsilon_{1}, \varepsilon_{1}>0 ; \\
& L_{4}: \quad \varepsilon_{20}=\frac{d_{0}\left(a+c_{0}\right)}{a\left(d_{0}+b\right)} \varepsilon_{1}, \varepsilon_{1}>0, L_{5}: \varepsilon_{20}=\frac{c_{0}}{a} \varepsilon_{1}, \varepsilon_{1}>0 .
\end{aligned}
$$

Now we consider the influence of phase angle $\theta$ to the map (15). By making a comparison between the map (16) and normal form map, we can unfold qualitative analyses for the normal form map. The fixed point $Y_{1}^{*}$ of the map (16) corresponds to the invariant circle of the normal form map, which is associated with the fixed point $Y_{0}^{(1)}=(0,0,0)^{\mathrm{T}}$. The fixed points $Y_{2 i}^{*}$ of the map (16) correspond to period two fixed points of the map (10). The fixed points $Y_{3 i}^{*}$ of the map (16) correspond to the invariant circles associated with the period two fixed points for the map (10). For the normal form map in the simple Cases (I) and (II), Hopf and period-doubling bifurcations associated with the fixed point $Y_{0}^{(1)}$ occur as parameters crossing the lines $L_{4}$ and $L_{1}$ from the region $\mathrm{R}_{1}$, respectively; crossing the line $L_{2}$ Hopf bifurcation associated with period two fixed points $Y_{i}^{(2)}$ occurs. The Hopf bifurcation associated with the fixed point $Y_{0}^{(1)}$ is supercritical for the Case (I), subcritical for the Case (II). For the normal form map in the complex Case (III), crossing the lines $L_{2}$ and $L_{1}$ Hopf and period-doubling bifurcations associated with the fixed point $Y_{0}^{(1)}$ occur, respectively; as the parameters crossing the line $L_{3}$ from the region $R_{2}$, the closed circle becomes non-attracting and the torus bifurcation occurs, which causes that the quasi-periodic attractor represented by two attracting closed circles is born. The quasi-periodic attractor represented by two closed circles is attracting in region $R_{3}$, and non-attracting in region $R_{4}$. It is to be noted that the period-doubling bifurcation associated with the fixed point $Y_{0}^{(1)}$ is subcritical for the complex case.

In view of Jacobian matrixes (13), we can find that there exist the period two fixed points $Y^{(2)}=\left(Y_{1}^{(2)}, Y_{2}^{(2)}\right)^{\mathrm{T}}$ as $\varepsilon_{1} / a<0$. According to the unfolding of the simple Case (I), the bifurcation set of the normal form map (10), 

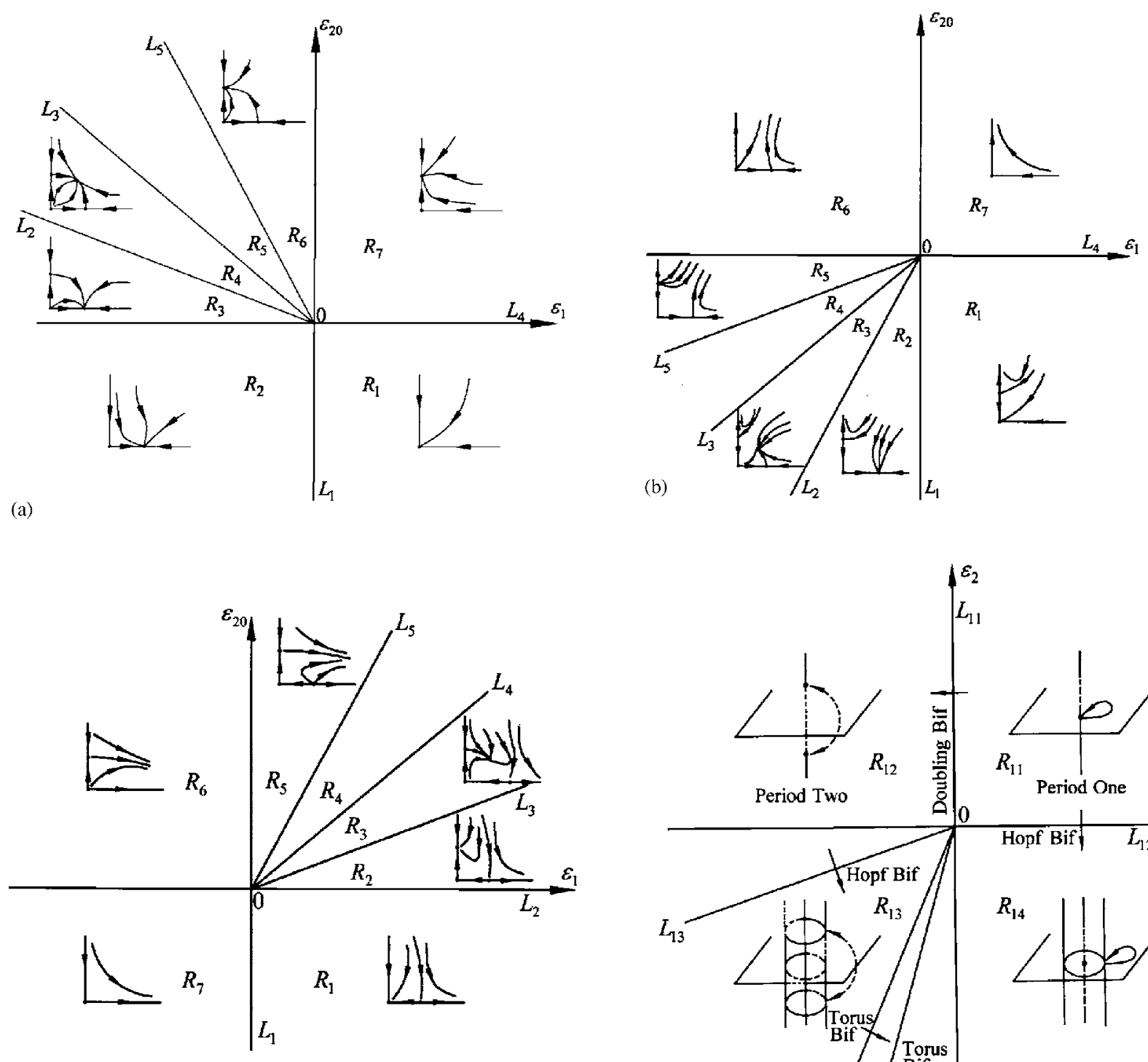

(c)

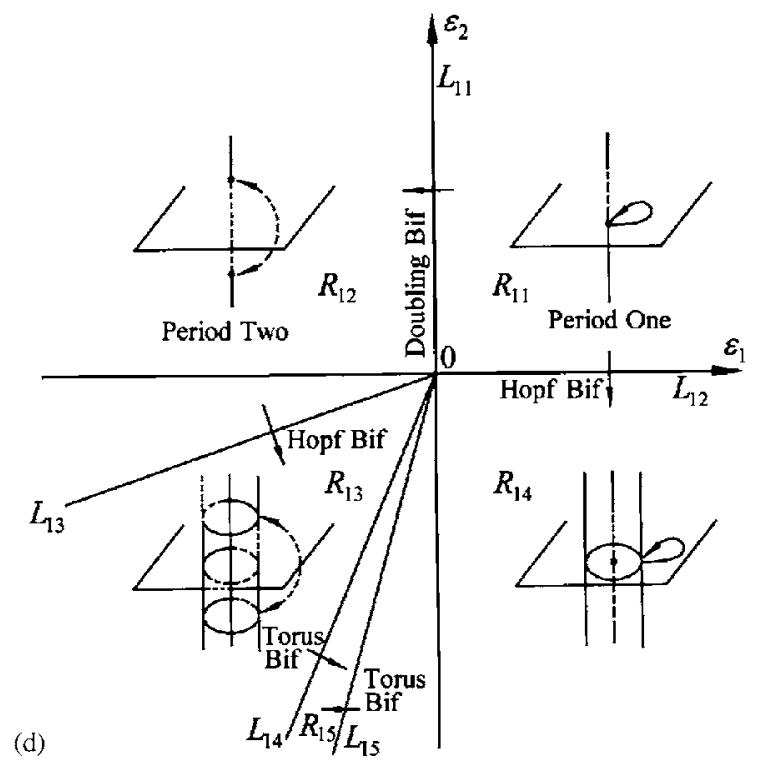

Fig. 2. The partial bifurcation sets of the simplified map and normal form map: (a) Case (I), the simplified map; (b) Case (II), the simplified map; (c) Case (III), the simplified map; (d) Case (I), the normal form map.

near $\varepsilon=(0,0,0)^{\mathrm{T}}$, can be further illustrated by Fig. 2(d) in which $\alpha<0$. The bounds of the regions shown in Fig. 2(d) can be listed as follow

$$
\begin{aligned}
& L_{11}: \varepsilon_{1}=0, \quad \varepsilon_{2}>0 ; \quad L_{12}: \varepsilon_{2}=0, \quad \varepsilon_{1}>0 ; \quad L_{13}: \varepsilon_{2}=\frac{e \varpi+c \alpha}{\alpha a} \varepsilon_{1}, \quad \varepsilon_{1}<0, \\
& L_{14}: \varepsilon_{2}=\frac{\alpha d+\varpi f}{\alpha b} \varepsilon_{1}, \quad \varepsilon_{1}<0 ; \quad L_{15}: \varepsilon_{2}=\frac{(\alpha d+\varpi f)(a+\alpha c+\varpi e)}{\alpha a(b+\alpha d+\varpi f)} \varepsilon_{1}, \quad \varepsilon_{1}<0,
\end{aligned}
$$

The bifurcation sets of the normal form map (10), associated with the Cases (II) and (III), can be obtained by using a similar method.

According to the center manifold theory, local behavior of map $f(v, X)$, near the bifurcation point $v_{\mathrm{c}}$, is equivalent to that of $\Phi(Y ; \varepsilon)$ for $\varepsilon$ near $\varepsilon=(0,0,0)^{\mathrm{T}}$. By virtue of the analyses of local bifurcation of the normal form map (10), 
we can find out dynamical behavior of the vibro-impact system in the case of Hopf-flip bifurcation. Local behavior of map $f(v, X)$, near the bifurcation point $v_{\mathrm{c}}$, conforms to the numerical results below.

\section{Numerical analyses}

In this section the analysis developed in the former section is verified by the presentation of results for the vibroimpact system. The existence and stability of $q=\frac{1}{1}$ motion are analyzed explicitly. Also, local bifurcations at the points of change in stability, discussed in the previous section, are considered, thus giving some information on dynamical behavior near the point of Hopf-flip bifurcation. The system, with parameters: $\mu_{m}=2.0, k_{1}=4.0, k_{3}=3.0$, $\delta=0.02, f_{20}=0$ and $R=0.7$, has been chosen for analysis. The forcing frequency $\omega$ and damping ratio $\zeta$ are taken as the control parameters, i.e. $v=(\zeta, \omega)^{\mathrm{T}}$. The eigenvalues of $\mathrm{D} f\left(v, X^{*}\right)$ are computed with $\omega \in[5.025,5.2]$ and $\zeta \in[0.01,0.02]$. All eigenvalues of $\mathrm{D} f\left(v, X^{*}\right)$ stay inside the unit circle for $v=(0.02,5.025)^{\mathrm{T}}$. By gradually increasing $\omega$ and decreasing $\zeta$ from the point $v=(0.02,5.025)^{\mathrm{T}}$ to change the control parameter $v$, we can obtain the eigenvalues $\lambda_{1}\left(v_{\mathrm{c}}\right)=-1.0000002$ and $\lambda_{2,3}\left(v_{\mathrm{c}}\right)=-0.3768488 \pm 0.9262742 \mathrm{i}\left(\left|\lambda_{2,3}\left(v_{\mathrm{c}}\right)\right|=0.9999994\right)$ which are very close to the unit circle, and the fourth eigenvalue $\left(\lambda_{4}\left(v_{\mathrm{c}}\right)=-0.4031064\right)$ still stays inside the unit circle as $v$ equals $v_{\mathrm{c}}=(0.0121275,5.074519)^{\mathrm{T}}$. The eigenvalues $\lambda_{1}(v)$ and $\lambda_{2,3}(v)$ have scaped the unit circle as $\omega$ (increasingly) and $\zeta\left(\right.$ decreasingly) pass through $v=(0.0121274,5.07452)^{\mathrm{T}}$. The eigenvalues $\lambda_{1}(v)$ and $\lambda_{2,3}(v)$ almost escape the unit circle simultaneously, so $v_{\mathrm{c}}=(0.0121275,5.074519)^{\mathrm{T}}$ may be approximately taken as the value of Hopf-flip bifurcation.

Local behavior of the vibro-impact system, near the point of Hopf-flip bifurcation, is obtained by numerical simulation. The partial bifurcation set near the critical value is plotted in Fig. 3. The bifurcation diagrams are shown for a series of values of $\zeta$ in Fig. 4. There exist two windows of $q=\frac{1}{1}$ motion in Figs. 4(a), (b) and (c), and only the second one occurs near the value of Hopf-flip bifurcation. It should be noted that the second region of forcing frequency associated with $q=\frac{1}{1}$ motion is narrow, and vanishes for $\zeta<0.0121275$ (see Figs. 4(d) and (e)). With increase in damping ratio $\zeta(\zeta>0.0121275)$, the second window of $q=\frac{1}{1}$ motion becomes wide gradually; see Figs. 4(a)-(c). The $q=\frac{1}{1}$ motion, associated with the first window, will undergo Hopf bifurcation with increase in $\omega$; see Fig. 4 . In the second region of forcing frequency associated with $q=\frac{1}{1}$ motion, the motion will undergo period doubling bifurcation with increase in $\omega$, and $q=\frac{2}{2}$ motion stabilizes. And then Hopf bifurcation of $q=\frac{2}{2}$ motion occurs so that the system exhibits the quasi-periodic impact motion associated with $q=\frac{2}{2}$ motion. The transition process of $q=\frac{1}{1}$ motion is plotted locally in an amplified form in Fig. 4(a1). The results from simulation show that no period doubling cascade of $q=\frac{1}{1}$ motion occurs, near the point of Hopf-flip bifurcation, due to occurrence of Hopf bifurcation of $q=\frac{2}{2}$ motion. Moreover, subcritical Hopf bifurcation of $q=\frac{1}{1}$ motion, in the second window, occurs with decrease in $\omega$ as seen in Figs. 4(a), (b) and (c), and the type of $q=\frac{1}{1}$ fixed point, from stable focus to unstable focus, is changed. Some projected Poincaré sections are plotted in Figs. 5-7. A representative analysis is given by choosing the damping ratio $\zeta=0.01225$ and changing the forcing frequency $\omega$ in numerical analysis. The numerical results show that the system exhibits stable $q=\frac{1}{1}$ motion with $\omega \in(5.073489,5.074559)$ as seen Fig. 3 and Fig. 4(a1). The $q=\frac{1}{1}$ motion undergoes

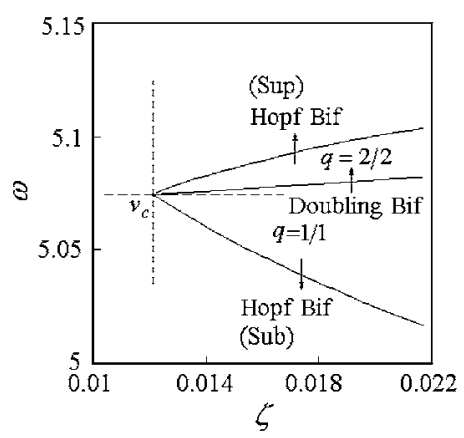

Fig. 3. The partial bifurcation set near the point of codim 2 bifurcation. 

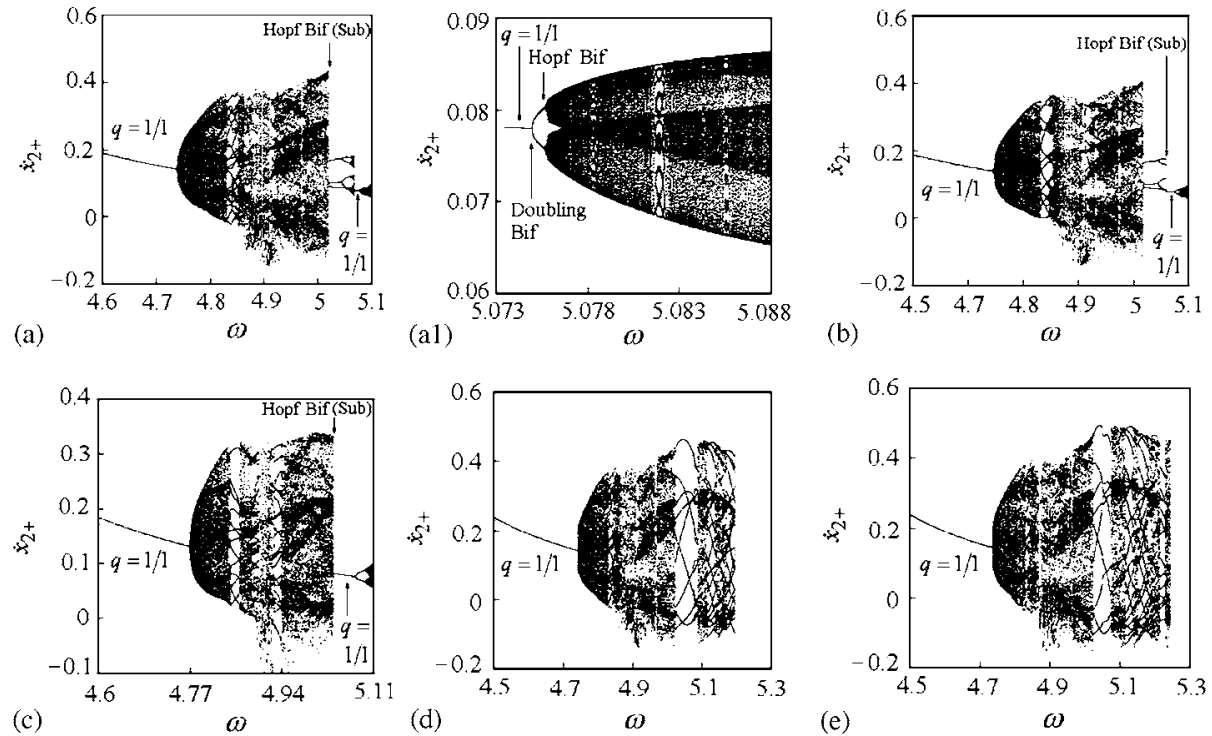

Fig. 4. Bifurcation diagrams near the point of codim 2 bifurcation: (a) $\zeta=0.01225$; (a1) $\zeta=0.01225$; (b) $\zeta=0.0135$; (c) $\zeta=0.0175$; (d) $\zeta=0.012$; (e) $\zeta=0.007$.
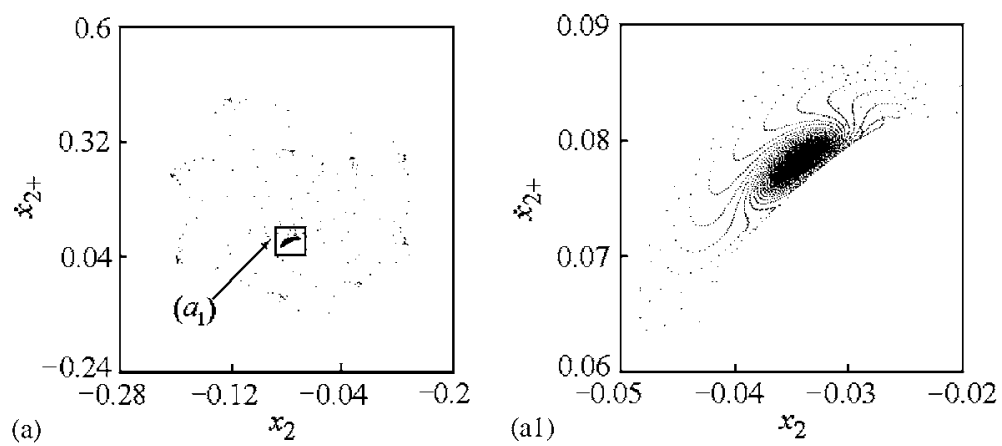

Fig. 5. Projected Poincaré sections: (a) transient points as well as $q=\frac{9}{9}$ fixed points, starting from the initial condition near the fixed point of $q=\frac{1}{1}$ motion (unstable focus), $\omega=5.0724, \zeta=0.01225$; (a1) local map near the unstable $q=\frac{1}{\mathrm{~T}}$ point, $\omega=5.0724, \zeta=0.01225$.

Hopf bifurcation as $\omega$ passes through $\omega_{\mathrm{c} 1}=5.073489$ decreasingly. The eigenvalues of $\mathrm{D} f\left(\omega, X^{*}\right)$ are given for $\omega_{\mathrm{c} 1}$ as follows

$$
\begin{aligned}
& \lambda_{1,2}\left(\omega_{\mathrm{c} 1}\right)=-0.3773860 \pm 0.9260564 \mathrm{i}, \quad\left|\lambda_{1,2}\left(\omega_{\mathrm{c} 1}\right)\right|=1.0000001, \quad \lambda_{3}\left(\omega_{\mathrm{c} 1}\right)=-0.9876892 \\
& \lambda_{4}\left(\omega_{\mathrm{c} 1}\right)=-0.4073028
\end{aligned}
$$

We can conclude, by the result from simulation, that subcritical Hopf bifurcation of $q=\frac{1}{1}$ motion occurs for $\omega<5.073489$, and the type of $q=\frac{1}{1}$ fixed point, from stable focus to unstable focus, is changed; see Fig. 5 which shows transient points as well as stable $q=\frac{9}{9}$ fixed points, starting from the initial condition near the unstable fixed point of $q=\frac{1}{1}$ motion for $\omega=5.0724$. The Period doubling bifurcation of $q=\frac{1}{1}$ motion occurs as $\omega$ is increased gradually and passes through $\omega_{\mathrm{c} 2}=5.074559$, and the system exhibits stable $q=\frac{2}{2}$ motion; see Fig. 4(a1). The $q=\frac{2}{2}$ motion changes its stability for $\omega>5.07585$, and Hopf bifurcation of $q=\frac{2}{2}$ motion occurs so that the system exhibits quasi-periodic impact motion associated with $q=\frac{2}{2}$ points as seen in Figs. 6(a) and (b). With further increase in $\omega$, the closed circle becomes quasi-attracting. The quasi-attracting invariant circle is attracting for the map point inside 

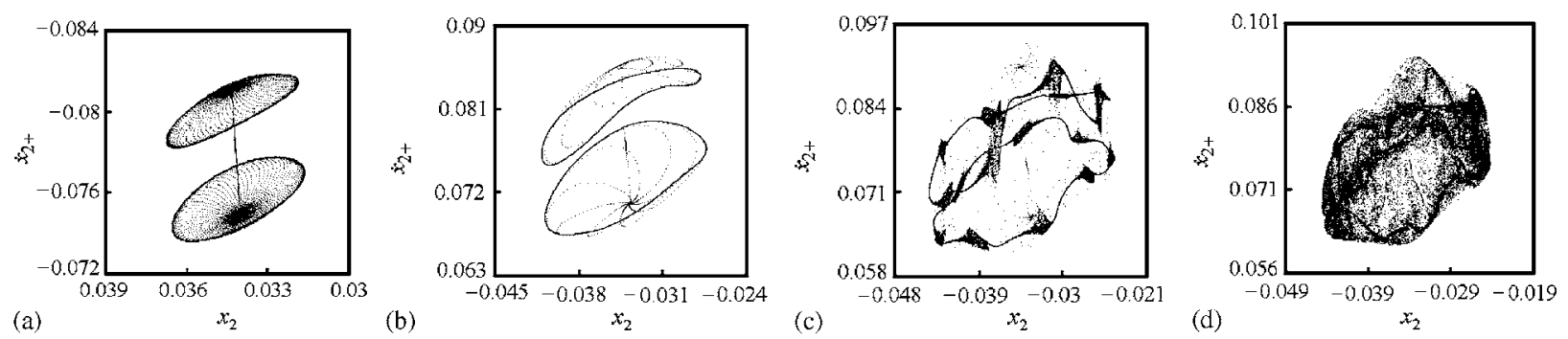

Fig. 6. Projected Poincaré sections: (a) attracting invariant circles of $q=\frac{2}{2}$ points, $\omega=5.076, \zeta=0.01225$; (b) attracting invariant circles of $q=\frac{2}{2}$ points, $\omega=5.083, \zeta=0.01225$; (c) chaos, $\omega=5.095, \zeta=0.01225$; (d) chaos, $\omega=5.096, \zeta=0.01225$.
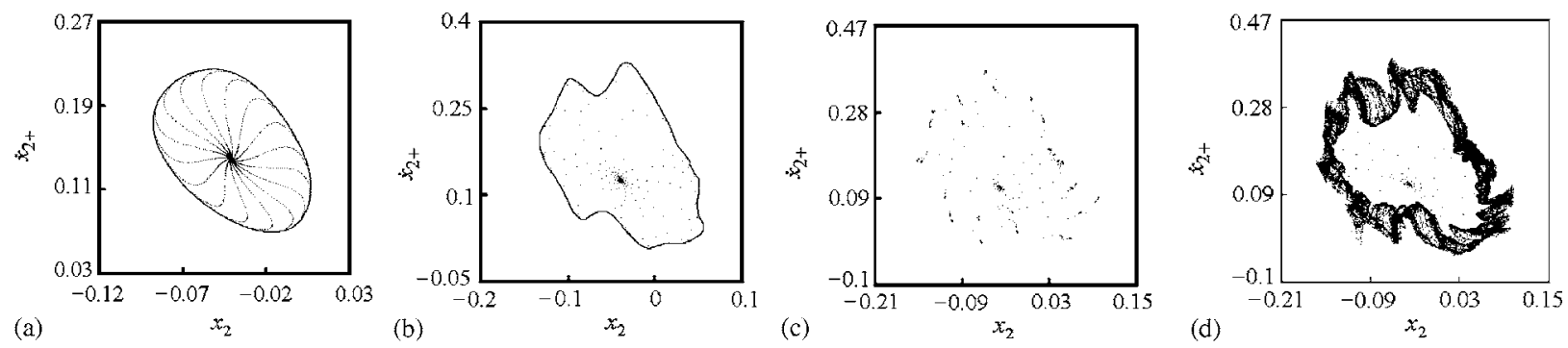

Fig. 7. Projected Poincaré sections: (a) attracting invariant circle of $q=\frac{1}{\mathrm{~T}}$ point, $\omega=4.75$; (b) attracting invariant circle of $q=\frac{1}{1}$ point, $\omega=4.8$; (c) phase locking, $\omega=4.85$; (d) chaos, $\omega=4.855$.

the circle, and repelling for the map point on or outside it. The system falls into chaotic motion immediately via the quasi-attracting invariant circle; see Figs. 6(c) and (d).

The $q=\frac{1}{1}$ motion, associated with the first window, undergoes Hopf bifurcation as $\omega$ passes through $\omega_{\mathrm{c} 3}=4.74017$ increasingly. With increase in the forcing frequency $\omega$, the quasi-periodic attractor transits to chaos via phase locking; see Fig. 7.

The dynamic behavior near the point of Hopf-flip bifurcation, occurring in the system of Fig. 1, corresponds with the unfolding of the simple Case (II) shown in Fig. 2(b).

In order to observe Hopf-flip bifurcations and transition phenomena of periodic-impact motions associated the complex case, we consider a vibro-bouncing system shown in Fig. 8. A body with mass $m$ bounces on the flat horizontal surface of a vibro-bench with masses $M_{1}$ and $M_{2}$. Displacements of these masses $m, M_{1}$ and $M_{2}$ are represented by $Y_{0}, X_{1}$ and $X_{2}$, respectively. The masses $M_{1}$ and $M_{2}$ are connected to linear springs with stiffnesses $K_{1}$ and $K_{2}$, and linear viscous dashpots with damping constants $C_{1}$ and $C_{2}$. The excitations on both masses of the vibro-bench are harmonic with amplitudes $P_{1}$ and $P_{2}$. The mass $M_{1}$ impacts mutually with the bouncing mass $m$ when they are on the same height, so the mass $m$ exhibits the bouncing motion.

The motion processes of the system, between any two consecutive impacts, are considered. Between consecutive impacts, the non-dimensional differential equations of motion are given by

$$
\begin{aligned}
& {\left[\begin{array}{cc}
1 & 0 \\
0 & \mu_{m}
\end{array}\right]\left\{\begin{array}{l}
\ddot{x}_{1} \\
\ddot{x}_{2}
\end{array}\right\}+\left[\begin{array}{cc}
2 \zeta & -2 \zeta \\
-2 \zeta & 2 \zeta\left(1+\mu_{\mathrm{c}}\right)
\end{array}\right]\left\{\begin{array}{l}
\dot{x}_{1} \\
\dot{x}_{2}
\end{array}\right\}+\left[\begin{array}{cc}
1 & -1 \\
-1 & 1+\mu_{k}
\end{array}\right]\left\{\begin{array}{l}
x_{1} \\
x_{2}
\end{array}\right\}=\left\{\begin{array}{c}
1-f_{20} \\
f_{20}
\end{array}\right\} \sin (\omega t+\tau),} \\
& \ddot{y}=-\beta,
\end{aligned}
$$

where the non-dimensional quantities are given by

$$
\mu_{m}=\frac{M_{2}}{M_{1}}, \quad \mu_{k}=\frac{K_{2}}{K_{1}}, \quad \mu_{\mathrm{c}}=\frac{C_{2}}{C_{1}}, \quad f_{20}=\frac{P_{2}}{P_{1}+P_{2}}, \quad \omega=\Omega \sqrt{\frac{M_{1}}{K_{1}}}, \quad t=T \sqrt{\frac{K_{1}}{M_{1}}},
$$




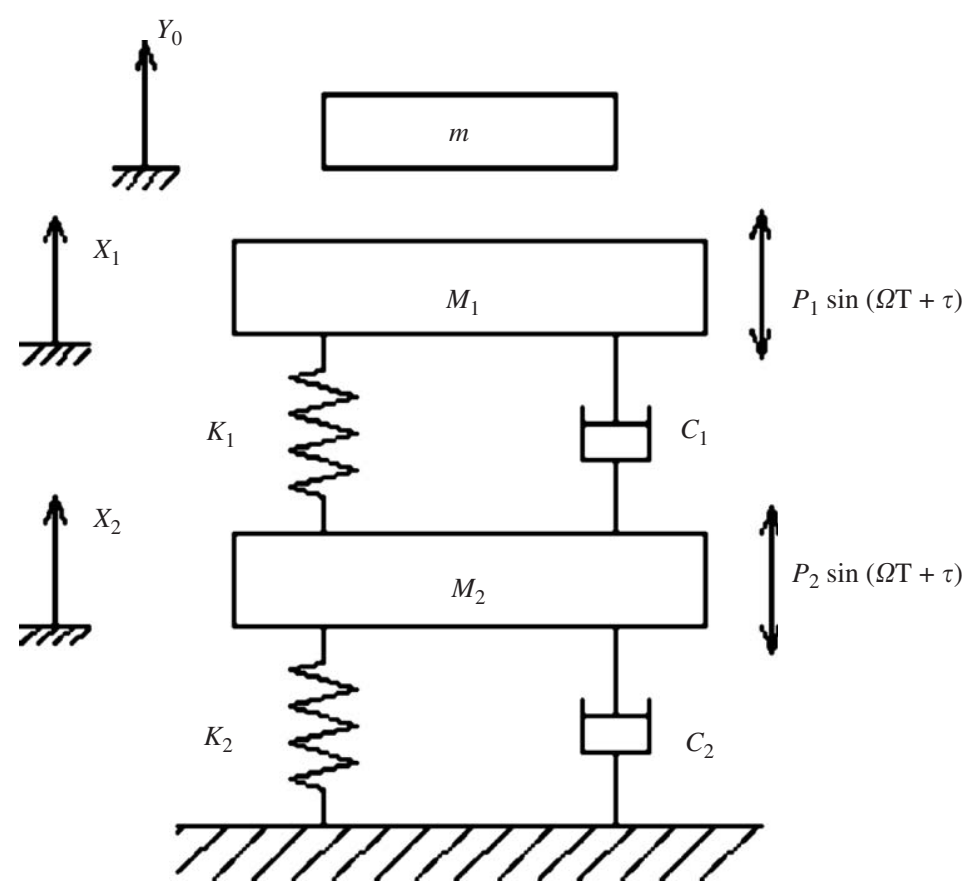

Fig. 8. Schematic of the vibro-impact system.

$$
\zeta=\frac{C_{1}}{2 \sqrt{K_{1} M_{1}}}, \quad x_{i}=\frac{X_{i} K_{1}}{P_{1}+P_{2}}, \quad \beta=\frac{M_{1} g}{P_{1}+P_{2}}, \quad \mu=\frac{m}{M_{1}}, \quad y=\frac{Y_{0} K_{1}}{P_{1}+P_{2}} .
$$

When the impact occurs, for $x_{1}(t)=y(t)$, the velocities of masses $m$ and $M_{1}$ are changed according to the conservation law of momentum, and the impact equation of both masses $m$ and $M_{1}$ and the coefficient of restitution $R$ are given by

$$
\dot{x}_{1-}+\mu \dot{y}_{-}=\dot{x}_{1+}+\mu \dot{y}_{+}, \quad \dot{x}_{1+}-\dot{y}_{+}=-R\left(\dot{x}_{1-}-\dot{y}_{-}\right) .
$$

where the subscript minus sign denotes the states just before impact and the subscript plus sign denotes the states just after impact, the velocities of two masses $M_{1}$ and $m$, just immediately before and after the impact, are represented respectively by $\dot{x}_{1-}, \dot{y}_{-}, \dot{x}_{1+}$ and $\dot{y}_{+}$.

A Poincaré section $\sigma=\left\{\left(x_{1}, \dot{x}_{1}, x_{2}, \dot{x}_{2}, y, \dot{y}, \theta\right) \in \boldsymbol{R}^{6} \times \boldsymbol{S}, x_{1}=y, \dot{x}_{1}=\dot{x}_{1+}, \dot{y}=\dot{y}_{+}\right\}$is chosen to establish Poincaré map of the impact system, which can be derived analytically from the equations of motion. The disturbed map of period one single-impact motion is expressed by

$$
X^{\prime}=\tilde{f}(v, X),
$$

where $X \in \boldsymbol{R}^{6}, v \in \boldsymbol{R}^{1}$ or $\boldsymbol{R}^{2}$ is varying parameter; $X=X^{*}+\Delta X, X^{\prime}=X^{*}+\Delta X^{\prime}, \Delta X=\left(\Delta \dot{x}_{1+}, \Delta x_{1}, \Delta \dot{x}_{2}, \Delta x_{2}, \Delta \dot{y}_{+}, \Delta \tau\right)^{\mathrm{T}}$ and $\Delta X^{\prime}=\left(\Delta \dot{x}_{1+}^{\prime}, \Delta x_{1}^{\prime}, \Delta \dot{x}_{2}^{\prime}, \Delta x_{2}^{\prime}, \Delta \dot{y}_{+}^{\prime}, \Delta \tau^{\prime}\right)^{\mathrm{T}}$ are the disturbed vectors of $X^{*}, X^{*}=\left(\dot{x}_{1+}, x_{10}, \dot{x}_{2}, x_{20}, \dot{y}_{+}, \tau_{0}\right)^{\mathrm{T}}$ is a fixed point in the hyperplane $\sigma$.

An interest torus doubling bifurcation is found to exist in the vibro-impact system near the point of Hopf-flip bifurcation associated with the complex case. The torus doubling bifurcation makes the quasi-periodic attractor associated with $q=\frac{1}{1}$ motion transit to the other quasi-periodic attractor represented by two attracting closed circles. The phenomenon concerning torus doubling bifurcation is demonstrated by the example in the following text.

The vibro-bounce system, with parameters: $\mu=0.3, \mu_{k}=1, \mu_{\mathrm{c}}=1, \beta=0.5, f_{20}=0, \zeta=0.05$ and $R=0.6$, has been chosen for analysis. The forcing frequency $\omega$ and distribution of masses $\mu_{m}$ are taken as the control parameters, i.e. $v=\left(\mu_{m}, \omega\right)^{\mathrm{T}}$. The eigenvalues of Jacobian matrix $\mathrm{D} \tilde{f}\left(v, X^{*}\right)$ are computed. All eigenvalues of $\mathrm{D} \tilde{f}\left(v, X^{*}\right)$ stay inside the unit circle for $v=(0.72,0.5275)^{\mathrm{T}}$. By gradually decreasing $\mu_{m}$ and $\omega$ from the point $v=(0.72,0.5275)^{\mathrm{T}}$ to change the control parameters $v$, we obtain the critical value $v_{\mathrm{c}}=(0.7084388,0.5275136)^{\mathrm{T}}$, at which all eigenvalues 


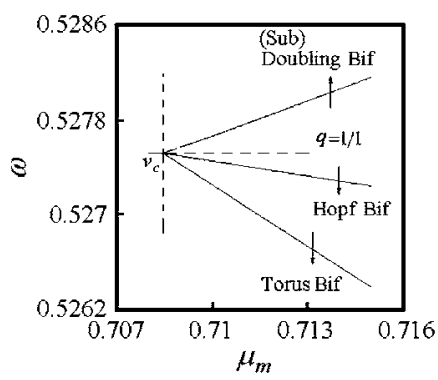

Fig. 9. The partial bifurcation set near the point of codim 2 bifurcation.
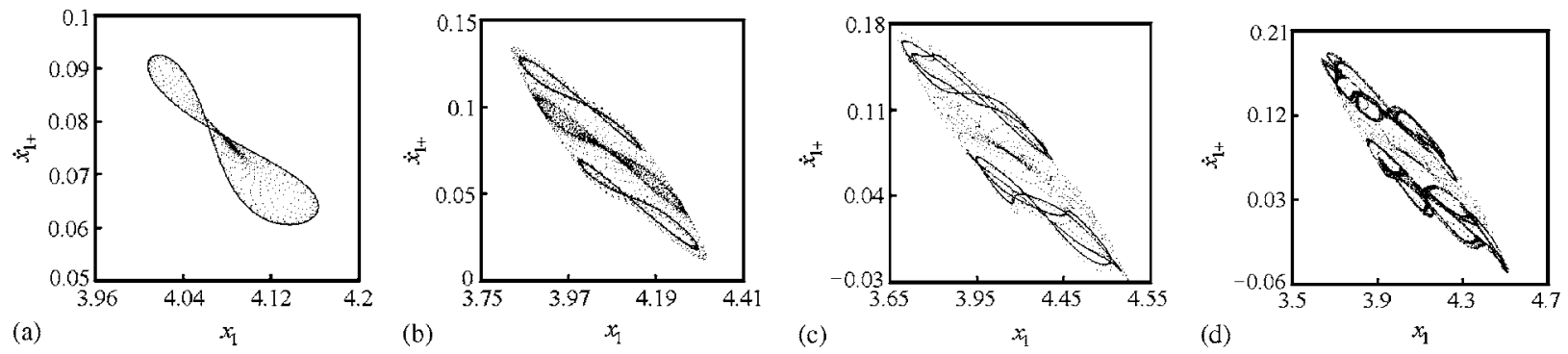

Fig. 10. Projected Poincaré sections: (a) attracting invariant circle of $q=\frac{1}{1}$ point, $\omega=0.5275$; (b) transient points, the non-attracting invariant circle as well as two attracting invariant circles caused by torus bifurcation, starting from the initial condition near the fixed point of $q=\frac{1}{1}$ motion, $\omega=0.5274$; (c) tori doubling, $\omega=0.52723$; (d) chaos, $\omega=0.52715$.

of Jacobian matrix $\mathrm{D} \tilde{f}\left(v, X^{*}\right)$ are given by

$$
\begin{aligned}
& \lambda_{1,2}\left(v_{\mathrm{c}}\right)=-0.28803001 \pm 0.95762230 \mathrm{i}, \quad\left|\lambda_{1,2}\left(v_{\mathrm{c}}\right)\right|=1.0000, \quad \lambda_{3}\left(v_{\mathrm{c}}\right)=-0.99999874 \\
& \lambda_{4}\left(v_{\mathrm{c}}\right)=-0.1849545, \quad \lambda_{5,6}\left(v_{\mathrm{c}}\right)=-0.11229740 \pm 0.08878266 \mathrm{i}
\end{aligned}
$$

The eigenvalues $\lambda_{1,2}\left(v_{\mathrm{c}}\right)$ and $\lambda_{3}\left(v_{\mathrm{c}}\right)$ are very close to the unit circle. As $v$ passes through $v=(0.7084387,0.5275134)^{\mathrm{T}}$ decreasingly the eigenvalues $\lambda_{1,2}(v)$ and $\lambda_{3}(v)$ have escaped the unit circle. So $v_{\mathrm{c}}=(0.7084388,0.5275136)^{\mathrm{T}}$ is approximately taken as the value of Hopf-flip bifurcation.

The partial bifurcation set near the critical value is plotted in Fig. 9. Numerical results are shown for $\mu_{m}=0.70845$ and a series of values of $\omega$ in Fig. 10. The process of torus bifurcation can be observed clearly from the projected portraits of Poincaré map. As the forcing frequency $\omega$ passes through the critical value $\omega_{\mathrm{c} 1}=0.527511$ decreasingly, a complex conjugate pair eigenvalues of $\mathrm{D} \tilde{f}\left(\omega, X^{*}\right)$ escape the unit circle, and supercritical Hopf bifurcation associated with $q=\frac{1}{1}$ motion occurs. The quasi-periodic attractor is shown for $\omega=0.5275 \mathrm{in} \mathrm{Fig.} \mathrm{10(a).} \mathrm{With} \mathrm{decrease} \mathrm{in} \omega$, instability of the closed circle associated with $q=\frac{1}{1}$ point occurs, and the torus bifurcation leads to two attracting invariant circles; see Fig. 10(b). With further decrease in $\omega$, the two invariant circles become non-attracting and tori doubling are born, which subsequently becomes unstable and chaotic; see Figs. 10(c) and (d). As the forcing frequency $\omega$ passes through $\omega_{\mathrm{c} 2}=0.5275321$ increasingly, a real eigenvalue of Jacobian matrix $\mathrm{D} \tilde{f}\left(\omega, X^{*}\right)$ escapes the unit circle from the point $(-1,0)$, and period doubling bifurcation associated with $q=\frac{1}{1}$ motion occurs. However, the period doubling bifurcation is subcritical. Stable $q=\frac{1}{1}$ motion and unstable $q=\frac{2}{2}$ motion coexist in the region $\omega \in(0.527511,0.5275321)$. After $\omega$ passes through $\omega_{\mathrm{c} 2}=0.5275321$ increasingly, the $q=\frac{1}{1}$ motion becomes unstable and there exists no $q=\frac{2}{2}$ motion.

The dynamic behavior near the point of Hopf-flip bifurcation, occurring in the example of the system shown in Fig. 8, corresponds with the unfolding of the complex Case (III) shown in Fig. 2(c). 


\section{Conclusions}

Dynamical behavior of the vibro-impact systems, near the point of Hopf-flip bifurcation, is investigated by qualitative analyses and numerical simulation. The vibro-impact systems, under the condition of codim 2 bifurcation, can exhibit more complicated quasi-periodic impact motions than those which occur in non-resonance and weak resonance cases [13]. Near the point of Hopf-flip bifurcation there exists not only Hopf bifurcation of $q=\frac{1}{1}$ motion, but also Hopf bifurcation of $q=\frac{2}{2}$ motion or torus bifurcation. The period doubling bifurcation of $q=\frac{1}{1}$ motion is commonly existent for the simple cases near the point of Hopf-flip bifurcation. However, no period doubling cascade of the motion emerges due to occurrence of Hopf bifurcation of $q=\frac{2}{2}$ motion. The $q=\frac{2}{2}$ fixed points are symmetrical about the corresponding $q=\frac{1}{1}$ point. The results from simulation show also that the attracting invariant circles associated with $q=\frac{2}{2}$ points are smooth in nature and symmetrical about the corresponding $q=\frac{1}{1}$ point near the value of Hopf bifurcation of $q=\frac{2}{2}$ fixed points. As the value of $\omega$ moves further away from the value of Hopf bifurcation, two attracting invariant circles expand, and the smoothness and symmetry of the quasi-periodic attractor are changed by degrees until they are destroyed.

An interest torus doubling bifurcation is found to exist near the value of Hopf-flip bifurcation. The torus doubling bifurcation makes the quasi-periodic attractor associated with period one single-impact motion transit to the other quasi-periodic attractor represented by two attracting closed circles. The torus bifurcation is qualitatively different from the typical torus doubling bifurcation occurring in the vibro-impact systems.

The strict condition of codimension two bifurcation is not easy to encounter in practical application of engineering. However, there exist the possibilities that actual nonlinear dynamical systems, with two varying parameters or more, work near the critical value of codim 2 bifurcation due to change of parameters. The impact-forming machinery is a typical example. Besides the forcing frequency $\omega$, the clearance varies also with different thickness of the formed workpieces [22]. Another representative example is the inertial shaker, of which the distribution of masses is generally metabolic with the casts with different masses, and the forcing frequency is also important parameter changed [21]. The change of multi-parameters possibly leads to the results that the vibro-impact systems work near the critical parameters of codim 2 bifurcation. It is necessary to study the bifurcations caused by change of multi-parameters and dynamical behavior of nonlinear systems near the points of bifurcations.

\section{Acknowledgements}

The author gratefully acknowledge the support by National Natural Science Foundation (10572055, 50475109), Natural Science Foundation of Gansu Province Government of China (ZS-031-A25-007-Z (key item)) and 'Qing Lan' Talent Engineering by Lanzhou Jiaotong University.

\section{References}

[1] J.O. Aidanpää, B.R. Gupta, Periodic and chaotic behaviour of a threshold-limited two-degree-of-freedom system, J. Sound Vib. 165 (2) (1993) 305-327.

[2] B. Balachandran, Dynamics of an elastic structure excited by harmonic and a harmonic impactor motions, J. Vib. Control 9 (3-4) (2003) 265-279.

[3] C. Budd, F. Dux, A. Cliffe, The effect of frequency and clearance variations on single-degree-of-freedom impact oscillators, J. Sound Vib. 184 (3) (1995) 475-502.

[4] X. De Souza, L.T. Silvio, I.L. Caldas, Calculation of Lyapunov exponents in systems with impacts, Chaos, Solitons and Fractals 19 (3) (2004) 569-579.

[5] M.I. Feigin, The increasingly complex structure of the bifurcation tree of a piecewise-smooth system, J. Appl. Math. Mech. 59 (6) (1995) 853-863.

[6] Q. Feng, H. He, Modeling of the mean Poincare map on a class of random impact oscillators, European J. Mech. A/Solids 22 (2) (2003) 267-281.

[7] B.D. Hassard, N.D. Kazarinoff, Y.H. Wan, Theory and applications of Hopf bifurcation, London Math. Society Lecture, Note Series, vol. 41, Cambridge University Press, Cambridge, 1981, pp. 86-95.

[8] Z.L. Huang, Z.H. Liu, W.Q. Zhu, Stationary response of multi-degree-of-freedom vibro-impact systems under white noise excitations, J. Sound Vib. 275 (1-2) (2004) 223-240.

[9] A.P. Ivanov, Stabilization of an impact oscillator near grazing incidence owing to resonance, J. Sound Vib. 162 (3) (1993) $562-565$.

[10] J. Knudsen, A.R. Massih, Dynamic stability of weakly damped oscillators with elastic impacts and wear, J. Sound Vib. 263 (1) (2003) 175-204. 
[11] R.I. Leine, D.H. Van Campen, Ch. Glocker, Nonlinear dynamics and modeling of various wooden toys with impact and friction, J. Vib. Control 9 (1-2) (2003) 25-78.

[12] G.W. Luo, Periodic motions and bifurcations of vibro-impact systems, Doctor's Degree Dissertation, Southwest Jiaotong University, 1998.

[13] G.W. Luo, J.H. Xie, Hopf bifurcation of a two-degree-of-freedom vibro-impact system, J. Sound Vib. 213 (1998) $391-408$.

[14] C.J. Luo Albert, An unsymmetrical motion in a horizontal impact oscillator, J. Vib. Acous. Trans. ASME 124 (3) (2002) $420-426$.

[15] C.J. Luo Albert, Dynamic stability of weakly damped oscillators with elastic impacts and wear, Chaos, Solitons Fractals 19 (4) (2004) 823-839.

[16] Y.V. Mikhlin, Direct and inverse problems encountered in vibro-impact oscillations of a discrete system, J. Sound Vib. 216 (2) (1998) 227-250.

[17] K.D. Murphy, T.M. Morrison, Grazing instabilities and post-bifurcation behavior in an impacting string, J. Acoust. Soc. Am. 111 (2) (2002) $884-892$.

[18] A.B. Nordmark, Non-periodic motion caused by grazing incidence in an impact oscillator, J. Sound Vib. 145 (2) (1991) $279-297$.

[19] F. Peterka, J. Vacik, Transition to chaotic motion in mechanical systems with impacts, J. Sound Vib. 154 (1) (1992) $95-115$.

[20] D.J. Wagg, Rising phenomena and the multi-sliding bifurcation in a two-degree of freedom impact oscillator, Chaos, Solitons and Fractals 22 (3) (2004) 541-548.

[21] B.C. Wen, Y.L. Li, Q.K. Han, Theory and Application of Nonlinear Oscillation, Northeast University Press, Shenyang, 2001.

[22] B.C. Wen, F.Q. Liu, Theory and Application of Vibratory Mechanism, Mechanism Industry Press, Beijing, 1992.

[23] K. Yu, C.J. Luo Albert, The periodic impact responses and stability of a human body in a vehicle traveling on rough terrain, J. Sound Vib. 272 (1-2) (2004) 267-286. 